\title{
Dario D’Incerti
}

La definizione delle cosiddette "competenze manageriali" è stata, ed è tuttora, oggetto di innumerevoli studi e ricerche e ha sostanzialmente accompagnato il progressivo aumento della complessità, reale o percepita, delle organizzazioni contemporanee. Di fatto, la risposta alle sfide della modernità ha comportato la necessità di adeguare una classe dirigente cresciuta secondo modelli datati e, attraverso una minuziosa descrizione delle sue capacità, abilità, skills..., metterla in condizioni di assolvere più efficacemente ai propri compiti. Il manager oggi deve essere capace di galleggiare nelle contraddizioni della sua epoca, deve essere innovativo e rispettoso delle tradizioni, con lo slancio della gioventù e la saggezza dell'esperienza, capace di affrontare il noto e l'ignoto, decisionista ma anche capace di ascolto, dotato di sufficiente autostima ma anche in grado di valorizzare i collaboratori. E così via. È accaduto quindi che la quantità di cose che il manager dovrebbe essere in grado di fare è aumentata a dismisura ed è divenuto centrale il bisogno di riflettere sui processi di apprendimento di tutte queste competenze e individuarne i relativi strumenti di valutazione. Si è poi insistito sul fatto che la formazione del manager non può, di fatto, mai avere fine ed è altresì indispensabile darsi ad attività di auto-formazione. Infine, si è dato spazio al concetto di meta-competenza, precondizione indispensabile perché avvenga un qualsiasi processo di formazione e di apprendimento. Non basta imparare, bisogna "imparare ad imparare". E bisogna anche - altra novità - capire che la componente razionale della conoscenza è sempre accompagnata da un sentimento, da uno stato emotivo che la connota e spesso la condiziona. Tutto ciò ha inevitabilmente allargato il campo degli strumenti attraverso cui favorire questi processi, andando "oltre l'aula" (come recitava il titolo di un libro di Boldizzoni e Nacamulli) e introducendo linguaggi a maggior contenuto emotivo come la letteratura, il teatro e il cinema. Il percorso che quest'ultimo ha fatto nei suoi centoventi anni di vita per ritagliarsi uno spazio significativo nel panorama degli artefatti culturali è stato tutt’altro che facile, e ancor più difficile è stato accreditarsi come strumento di conoscenza e apprendimento: ha dovuto prima di tutto contrastare la diffidenza che il mondo della pedagogia ha sempre avuto nei confronti delle innovazioni tecnologiche, viste spesso come fonti di alienazione (in una contrapposizione fra psichè e technè che a volte riaffiora anche oggi), e ha poi sofferto di una derubricazione a "pratica bassa", a espressione di una sottocultura di massa che lo assimilava ai beni di consumo come la musica leggera e il fumetto.

Già Walter Benjamin dovette lottare per sostenere l'idea che la fotografia potesse essere una forma d'arte e fin d'allora - giova ricordarlo - si fece largo in lui un'intuizione destinata a legittimare l'uso delle immagini tecniche, statiche o dinamiche, come strumenti di formazione. Secondo Benjamin, infatti, non si trattava solo di considerare la fotografia come arte ma, 
sinergie Vol. 35, N. 102, 2017

viceversa, l'arte come fotografia, nel senso che l'arte è una delle forme attraverso cui la società elabora un pensiero e un'immagine di sé. Da questo punto di vista, tutte le invenzioni narrative che l'uomo ha prodotto, da Omero a David Foster Wallace, dai graffiti rupestri ai film in 3D, sono potenti contenitori simbolici con cui noi ci relazioniamo - ieri come oggi - nella fondata ipotesi che il fatto che siano invenzioni non compromette minimamente la nostra capacità di creare relazioni "reali" con esse ${ }^{1}$. Per questo si può dire - oggi come ieri - che noi siamo abitati dalla realtà ma anche dall'immaginario. Ora, è indubbio che il cinema, avendo riassunto in sé tutte le precedenti forme d’arte (pittura, letteratura, musica, fotografia...) di questo immaginario ha costituito per più di un secolo la parte preponderante (con buona pace di coloro che ritengono che i nuovi media lo abbiano - in una misura che è però difficile stabilire - soppiantato). Ciò dipende in primo luogo dalle caratteristiche strutturali del cinema, dal suo raccontare la realtà con la realtà, dall'essere contemporaneamente "completamente oggettivo e completamente soggettivo" (Pasolini. 1977), dalla sua polisemia che fa sì che ogni film sia unico ma non sia mai univoco. E stato anche giustamente sostenuto che "il cinema contiene più di quanto lo spettatore possa ricevere e lo spettatore riceve più di quanto il cinema contenga" (Nepoti, 2004).

L'uso del cinema nelle aule della formazione - pratica peraltro più diffusa di quello che si crede comunemente - potrebbe essere fatto rientrare in quel fenomeno sociale che va sotto il nome di spettacolarizzazione. A sua volta, la spettacolarizzazione rientra nella cosiddetta società massmediale o società dell'informazione. Il concetto centrale - detto in maniera estremamente semplificata - è che la nostra conoscenza della realtà è sempre meno frutto di un rapporto diretto con essa ma, al contrario, sempre più di un rapporto, appunto, "mediato". La mediazione va fatalmente verso forme sempre più estreme di spettacolarizzazione. L'informazione quindi si spettacolarizza e, d’altra parte, lo spettacolo "forma". Cioè a dire: si sarebbe tentati di affermare che è la formazione che deve andare verso forme sempre più spettacolarizzate (anche perché le generazioni che si affacciano hanno sempre più fame di linguaggi spettacolari) ma è vero anche il contrario e cioè lo spettacolo è divenuto un agente formativo, se non altro nel senso che è formatore di mentalità. Lo spettacolo, e quindi il cinema, si fa in sostanza portatore di valori, modelli culturali, mode e modi di vivere che in maniera a volte palese a volte carsica, vengono assimilati nei nostri comportamenti quotidiani e determinano il nostro agire ben più profondamente di quanto noi stessi siamo disposti ad ammettere. Esporsi ad una qualche forma di spettacolo ha dunque una valenza formativa di per sé. Laltro aspetto importante è l'irrompere nelle aule - anche qui già da tempo, si pensi all'uso dei casi - della narratività. Si riconosce cioè il valore non di una ricostruzione oggettiva della realtà - peraltro impossibile -, ma di una realtà narrata il cui statuto ontologico non è meno perspicuo di quello che siamo abituati ad attribuire alla realtà tout court. La narrazione introduce a sua volte un elemento necessariamente legato alla sfera delle emozioni, emozioni di cui, come abbiamo già detto, è stato finalmente

1 "Perché la falsità dei termini non implica fatalmente quella della loro relazione" in Beckett S., Molloy, p. 120. 
riconosciuto il valore cognitivo. Ci si potrebbe chiedere a questo punto perché si debba attribuire al cinema un significato pedagogico particolare che lo stacca dalle altre forme di spettacolo. Il cinema non è più, da anni, l'unico mezzo "narrativo"; è stato affiancato prima dalla televisione, poi dai nuovi strumenti cosiddetti multimediali (anche il cinema è multimediale) o interattivi. La televisione, in particolare, ha portato via spettatori al cinema, questo è fuor di dubbio. Ha però avuto leffetto, essendo considerata una pratica bassa, di collocare il cinema in una fascia più alta, sia culturalmente che qualitativamente. Il cinema conserva dunque un'idea di autorialità che è appunto un termine che trascina con sé un'idea di autorevolezza. Diviene così un linguaggio "forte", in grado di penetrare forse più incisivamente nella mente dei discenti di quanto non siano più in grado di fare pratiche d'aula ancorate a modelli prevalentemente verbali. Ė altrettanto evidente come la presenza del cinema nelle pratiche formative si configuri come una forma di riutilizzo, avvicinandosi in tal modo alle innumerevoli esperienze artistiche che si possono ricondurre alla poetica del frammento o al concetto, appunto, di riutilizzo (dall'objet trouvé di Marcel Duchamp all'assemblage di Joseph Cornell, dal Trash alla Green Art) e che hanno avuto grande rilevanza anche nel cinema (si pensi al cosiddetto Found Footage o ad unopera cruciale come "Historie(s) du Cinema" di J.L. Godard). Inoltre ha avuto spesso l'effetto di operare una sorta di ri-significazione e di ri-contestualizzazione con esiti a volte sorprendenti. Ma cè di più. Fra le direzioni che questo riutilizzo di opere cinematografiche può prendere, ce nè una su cui vale la pena di riflettere. La si può definire un effetto di "ri-estetizzazione", intesa come capacità di sentire il mondo (che si contrappone alla "anestetizzazione" che è invece uno degli effetti generalmente attesi - se non voluti - dal sistema mass-mediale). Il cinema diviene così una pratica ri-estetizzante $\mathrm{e}$ favorisce quel processo di separazione e distinzione che sono i due termini con cui si traduce di solito il termine greco "krinein", termine da cui deriva fra l'altro l'italiano "critica": uscire dall'aula, fisicamente o metaforicamente, e vedere un film, in quanto separazione e distinzione, è già una forma (di) critica. E una posizione "critica" nei confronti degli artefatti mediali di cui è costituito il nostro panorama esperienziale è la pre-condizione indispensabile non solo per lauto-formazione ma prima di tutto per una cittadinanza del mondo consapevole e matura.

\section{Bibliografia}

BOLDIZZONI D., NACAMULLI R.C. (2011), Oltre l'aula, Apogeo Education, Milano.

NEPOTI R. (2004), L'illusione filmica. Manuale di filmologia, UTET, Torino.

PASOLINI P.P. (1977), Empirismo eretico, Garzanti, Milano.

\section{Academic or professional position and contacts}

Dario D'Incerti

Professionista della comunicazione

e-mail: sgarbi_dincerti@iol.it
Dario D'Incerti

Il valore del cinema per il

Management 